\title{
El multifamiliar contemporáneo en la manzana limeña: el caso de Magdalena del Mar
}

\author{
Moris Fleischman, Diego Franco, Héctor Loli, Jorge Sánchez \\ Universidad de Lima, Perú
}

Recibido: 29 de agosto del 2016 / Aprobado: 22 de diciembre del 2016

El artículo de investigación analiza la problemática en el diseño de los edificios de vivienda multifamiliar que se construyen en Lima. Actualmente, las dimensiones de los lotes, las normativas locales y la presión inmobiliaria producen unos tipos de edificios persistentes en su configuración. Pretendemos develar aquellos rasgos que están re-tipificando los tejidos urbanos tradicionales de la ciudad, como es el caso del distrito de Magdalena del Mar. La metodología se basa conceptualmente en un entendimiento tipológico de la vivienda, y operativamente en el uso del dibujo como herramienta de análisis. Finalmente, reflexionamos si estas contingencias permiten proyectar edificios con una idea colectiva de ciudad. La investigación deja abierta la oportunidad de utilizar sus conclusiones en la retroalimentación de los parámetros urbanísticos municipales.

edificio multifamiliar, vivienda, tipología, Lima

\section{The Contemporary Multifamily Architecture in Lima Blocks: the Case of Magdalena del Mar}

The research article analyzes the difficulties of design on several multifamily buildings under construction in Lima. Nowadays, the dimensions of the lots, the regulations in Lima and the real estate pressure have lead to some kinds of buildings with a persistent configuration. We try to reveal some of the essential characteristics which are deeply re-categorizing many of the traditional urban tissues of the city, such is the case of the district of Magdalena del Mar. The methodology is conceptually based on a typological understanding of housing, and it is operatively based on the use of orthographic drawing as an analysis tool. Finally, we consider whether these contingencies allow the projection of buildings with a collective idea of the city. The research leaves open the possibility to use its conclusions in the feedback of the municipal urban parameters.

multifamily building, housing, typology, Lima 


\section{INTRODUCCIÓN}

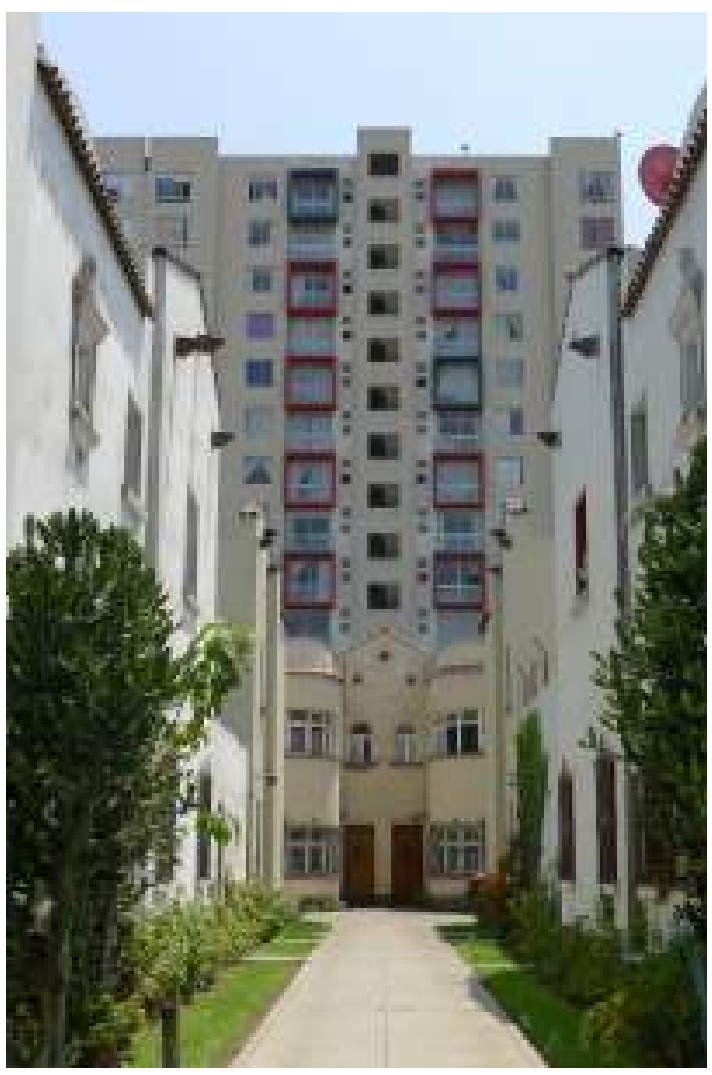

Figura 1

Verticalización del tejido tradicional limeño

Fotografía de los autores

El auge de la economía peruana en los últimos años, impulsada en gran medida por el sector de la construcción, trajo como consecuencia la paulatina transformación del paisaje urbano de Lima. El edificio de vivienda multifamiliar es la principal pieza arquitectónico-urbana de esta metamorfosis. ¿El resultado?: calles conformadas por infinidad de portones de garajes, enormes muros ciegos medianeros, ambientes interiores que miran a pozos minúsculos y grandes porcentajes de áreas libres que, paradójicamente, no pueden usarse de manera colectiva en las manzanas.

Aun cuando cerca del $70 \%$ de proyectos que se construyen formalmente en la capital son multifamiliares (Scotiabank, 2015, p. 7), es muy escasa la discusión informada y objetiva sobre este fenómeno, sus causas y consecuencias en la vida doméstica y colectiva. Por ello, la investigación se centra en el edificio de vivienda multifamiliar entre medianeras en la manzana limeña y toma como caso de estudio para el presente artículo una zona del distrito de Magdalena del Mar.

Proponemos una taxonomía de algunos ejemplos representativos para que, vistos y comparados en series o familias, nos permitan desmenuzar sus características tipológicas: circulaciones, dimensiones, agrupaciones, accesos, etcétera. A partir de la observación y el análisis, pretendemos inferir las consecuencias de estos rasgos en sus ocupantes y en la ciudad. ¿A quién están dirigidas las unidades de vivienda y qué condiciones de convivencia promueven? ¿Cuál es la visión de ciudad que construyen como conjunto?

Tanto el método operativo como las categorías propuestas servirán de herramientas para enriquecer críticamente el proceso proyectual de profesionales ligados al diseño y a la planificación de la vivienda urbana. Finalmente, las conclusiones obtenidas nos servirán de base para plantear mejoras en las normativas actuales de manera informada y propositiva, de cara a un futuro sostenible. 


\section{ESTADO DEL ARTE}

En el medio local existen pocas investigaciones que aborden el tema de la vivienda colectiva con un enfoque sistemático y analítico. En repetidas ocasiones, Ludeña (2002, 2006a, 2006b, 2011) ha teorizado sobre la influencia del contexto político y social en la evolución morfológica de Lima durante los siglos XIX y XX. A pesar de su rigor historiográfico y documental, echamos en falta una mayor profundización de las relaciones entre morfología urbana y tipología edilicia. Kahatt (2015) analiza ejemplos paradigmáticos de unidades vecinales, agrupaciones y conjuntos residenciales limeños. Si bien el autor aplica un análisis gráfico al hecho arquitectónico para comprobar interpretaciones, trata prototipos de vivienda colectiva ajenos al multifamiliar. Ciertamente, la arquitectura contemporánea en general y el edificio de vivienda multifamiliar entre medianeras en particular carecen de una profunda reflexión disciplinar.

Sin embargo, el estudio de la vivienda colectiva es un ámbito que ha cobrado enorme relevancia desde inicios del siglo xx. Cuando las ciudades europeas se enfrentaron al reto de construir viviendas adecuadas para las emergentes clases obreras, la arquitectura tuvo que tomar prestados de otros campos métodos de análisis y clasificación. Esta mirada científica permitió ordenar y comparar experiencias de distintos países en el marco de los Congresos Internacionales de Arquitectura Moderna (CIAM). Continuando con esta tradición, muchas publicaciones recientes (Mozas y Fernández-Per, 2004; Leupen y Mooij, 2011; Martín Blas, 2010) han sistematizado la manera que se estudian y se proyectan los edificios de vivienda colectiva. Los criterios de uniformización en la representación (dibujo) y en la cuantificación numérica (datos) son estándares que rescatamos para nuestro análisis comparativo.

\section{APROXIMACIÓN AL TIP0: TRABAJO EN SERIES}

La teoría arquitectónica ha complementado posteriormente el sesgo moderno por la técnica y la ciencia, abriendo el campo a lecturas cualitativas. Aldo Rossi (1999 [1971]) rescata el valor de aquellos edificios que en su repetición son capaces de tipificar determinadas extensiones urbanas. El uso de la idea del tipo para explicar la ciudad y su crecimiento ha sido retomado por Rafael Moneo (1978), quien sostiene que, como una canasta o una taza, el objeto arquitectónico no solo podía ser repetido, sino que estaba destinado a repetirse (p. 23). En este sentido, para Jacoby y Lee (2007), el tipo no puede ser entendido a través de un caso aislado pues este "es un objeto que pertenece a un grupo que comparte atributos similares" (p. 136). Así, para pensar y analizar tipológicamente es necesaria una clasificación en series o familias. Y la tipología, por otro lado, representa la forma de pensar y experimentar a través del tipo.

Para tratar de entender la ciudad a través de su morfología planteamos la taxonomía de una serie de ejemplos o tipos particulares, como una aproximación tipológica a la Lima del siglo XxI. Como Rossi, el mismo Lee sostiene que las áreas urbanas pueden ser descritas y conceptualizadas a través de su arquitectura más repetida, o lo que es lo mismo, sus “tipos dominantes".Y así como Manhattan puede ser entendida a través de la repetición de sus rascacielos, Londres a través de sus series de casas aterrazadas y Barcelona a través del conjunto de manzanas ochavadas, planteamos que el edificio de vivienda multifamiliar entre medianeras es un tipo de edificio que redefine los entornos urbanos de Lima y a su vez encarna el "significado simbólico, principios estructurales y lógica organizacional de la ciudad" (2007, p. 147). Es en la disección de estos edificios que podemos encontrar el ADN de muchas partes de la Lima contemporánea. 


\section{ARQUITECTURA ANÓNIMA}

La idea de entender, clasificar y teorizar la arquitectura común o anónima de la ciudad no es nueva. Desde el provocador libro Aprendiendo de Las Vegas, de Venturi, Izenour y Scott Brown (2004 [1972]), hasta el mismo Delirio de Nueva York, de Rem Koolhaas (2004 [1978]), muchos arquitectos han tratado de entender la ciudad y la vida urbana desde una mirada crítica a sus elementos más comunes, aquella arquitectura de fondo que en su repetición conforma el tejido urbano.

Dos publicaciones recientes siguen esta línea de pensamiento. Made in Tokyo, del grupo japonés Atelier Bow Wow (Kaijima, Kuroda, Tsukamoto, 2008), plantea una taxonomía de edificios urbanos híbridos o de programas de usos aparentemente incompatibles, cuya complejidad es resultante de necesidades inmediatas, cierta desregulación, y de ver la arquitectura sin prejuicios.Y Río Metropolitano (Lassance, Varella, Costa-Capille, 2013), que utiliza mecanismos muy similares para intentar disgregar las paradojas de una ciudad no menos compleja como Río de Janeiro.

El análisis comparativo y el uso del dibujo ortográfico como medio para adentrarse en la arquitectura son recursos compartidos por ambas publicaciones. Ciertamente constituyen referentes en fondo y forma de la investigación que da pie a este artículo.

\section{MATERIALES Y MÉTODOS}

El área urbana elegida abarca parte del distrito de Magdalena del Mar. Las vías que la demarcan son la avenida La Marina por el norte, la avenida Bertolotto por el sur, el jirón Larco Herrera por el este y el jirón Castilla por el oeste.

Ha sido seleccionada por dos razones. Primero, por su alto potencial de cambio en los próximos años. Ante la saturación y encarecimiento de otras áreas, el mercado inmobiliario ha volteado la mirada hacia zonas como esta, lo que facilita el estudio del tipo de edificio que pretendemos analizar. Segundo, por sus características morfológicas, tales como: 1. Homogeneidad en el tamaño de sus manzanas: el área está compuesta por la típica manzana cuadrada limeña $100 \mathrm{~m} \times 100 \mathrm{~m}$ que, a su vez, contiene lotes profundos $(10 \mathrm{~m}$ a $20 \mathrm{~m}$ de frente $\times 30 \mathrm{~m}$ a $40 \mathrm{~m}$ de fondo). 2. Edificios de altura: los parámetros normativos permiten alturas que oscilan entre los 7 y 15 pisos, que generan en estos tipos las mayores dificultades de diseño y morfológicos (Heckmann, Schneider, 2011, p. 77).

Los ejemplos elegidos se estudian comparativamente, sobre la base de las mismas categorías de clasificación y análisis, a fin de poder contraponer los edificios de forma neutral y directa. Con un código gráfico estándar, utilizamos el dibujo como herramienta analítica según diversas categorías agrupadas en dos escalas.

Por razones de extensión del artículo, comentaremos solo algunos casos de edificios y unidades dentro del área del estudio; sin embargo, la investigación incorpora además ejemplos fuera de Magdalena para enriquecer los resultados y sustentar las conclusiones. 


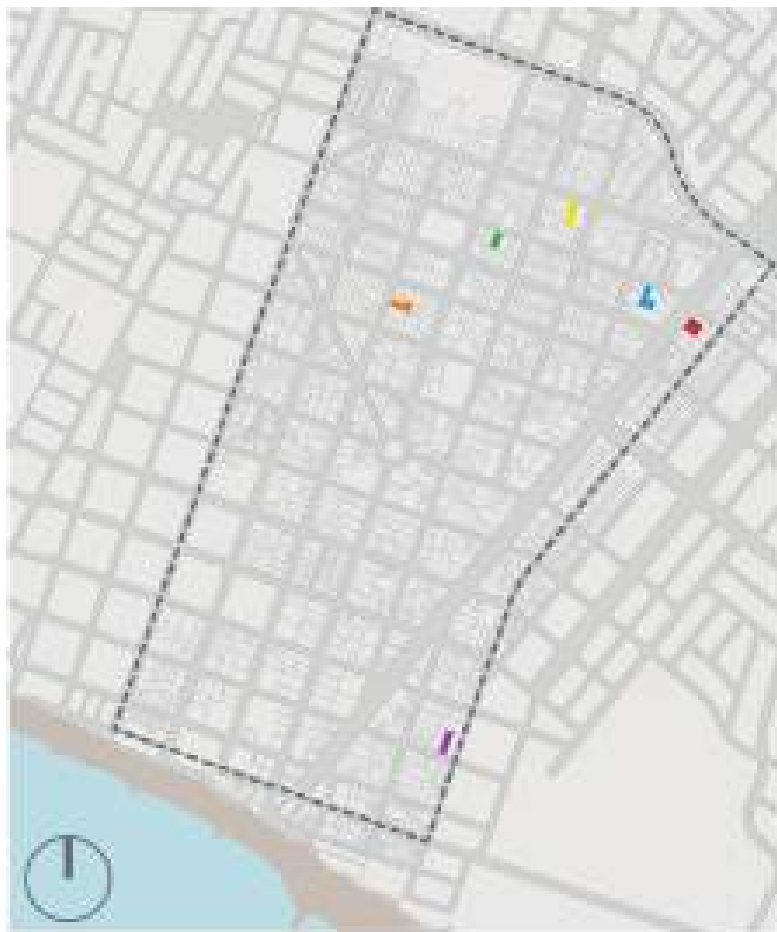

Tere Arezow II

Residencial Tacna

Edificio Las Acacias

Edificio Amaxomas

Residencial Dedas Odilis

Torres de Arica

Figura 2

El polígono demarca la extensión del área de estudio con su tejido urbano y los casos comentados Elaboración propia 


\section{EL EDIFICIO}

A esta escala se analiza la agrupación de las unidades como conjunto:el edificio multifamiliar. Se pretende comprender la resolución de dicho ensamble y resaltar las condiciones que hacen de la vivienda en vertical una experiencia de vida en comunidad, tanto en el interior como en su relación con la calle (Pérez de Arce, 2012).
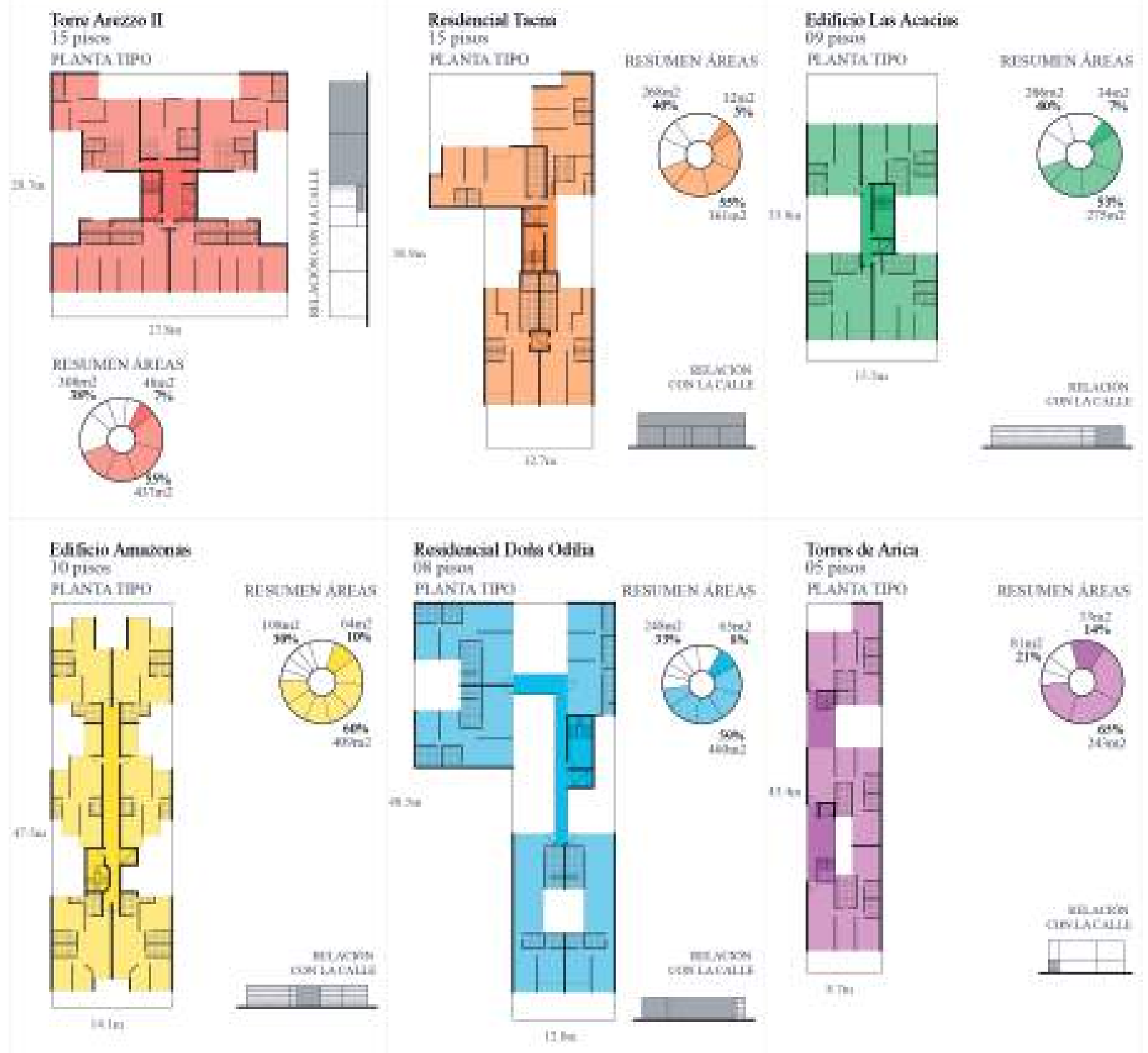

Figura 3

Dibujos de la planta típica a escala de edificio de los casos con sus respectivos gráficos de áreas y de relación con la calle Elaboración propia 
Resumen de áreas. En un gráfico tipo torta se distribuyen los porcentajes relativos destinados a cada tipo de área según las texturas utilizadas en el dibujo (áreas libres, áreas de circulación común y áreas de departamentos). Se complementa cada una indicando los valores absolutos en metros cuadrados. La relación entre ellos permite acercarse a la economía con la que se resuelve el edificio.

Subdivisión de área libre. La finalidad es visualizar la fragmentación del porcentaje de área libre requerido en el interior del lote. Según sus dimensiones y altura, se pretende inferir las condiciones lumínicas y de ventilación en el interior de los ambientes a los que sirven. También se puede determinar en qué medida este parámetro se convierte en un espacio horizontal útil para el edificio o si se trata de un pozo vertical para cumplir con las dimensiones mínimas que exige la norma.

Tipos de unidades. Esta clasificación se refiere a las variantes básicas agrupables según sus frentes libres: unidad de orientación simple, unidad de doble orientación y unidad de esquina (Sherwood, 1989). El uso de uno u otro tipo tiene implicancias decisivas en cuanto a sus posibilidades de ensamblaje y, por tanto, en el tipo de edificio que se configura. En un nivel más deductivo, la clasificación indica una posible diversidad en la convivencia de distintos modos de vida doméstica y habitantes en el edificio.

Agrupación y acceso. Debido a que no existe gran variedad en los tipos de edificios (bloque, torre, manzana, hilera, aislada, relleno) (Leupen y Mooji, 2011, p. 145) que admiten los lotes típicos, cobra mayor relevancia la caracterización detallada de la distribución hacia las unidades. Esta categoría define, al igual que los tipos de unidad, la manera en que la resolución del ensamble se acomoda a un lote y a parámetros específicos. El ratio entre cantidad de unidades por núcleo es uno de los principales indicadores de la economía del edificio. De forma inversa, un edificio poco compacto brinda la posibilidad de articular un itinerario de transición de la calle al departamento, que promueva el contacto entre sus ocupantes.

Cerramiento perimétrico. Compara relativamente la extensión del perímetro construido según tres condiciones de relación o transparencia: ciego, hacia el pozo y hacia la calle. Cada una se acompaña de su dimensión en metros lineales por planta, a fin de comparar la calidad y cantidad de superficie que se destina a la envolvente del edificio. No solo tiene que ver con la economía de su factor de forma, es decir, la relación entre superficie exterior y volumen interior, sino también con deducir la imagen de estos edificios hacia el exterior: el paisaje urbano que definen.

Relación con la calle. Mediante un dibujo simplificado de líneas y texturas del primer nivel en elevación se intenta definir la relación que tiene el edificio con la vereda y la manera en que inicia la transición hacia un espacio íntimo desde la calle, cómo se utiliza la premisa de retiro para lidiar con la transición entre la esfera pública y privada. La permeabilidad de las superficies utilizadas se señala con distintas densidades de texturas, así como los ingresos vehiculares y peatonales. 
LA UNIDAD

Del análisis a esta escala pretendemos inferir en qué condiciones las unidades que se diseñan responden a las nuevas demandas sociales, culturales y económicas de estructuras de convivencia variables en el tiempo. Aquello que Atelier Kempe Thill denomina "neutralidad específica” (2004, pp. 136-145).

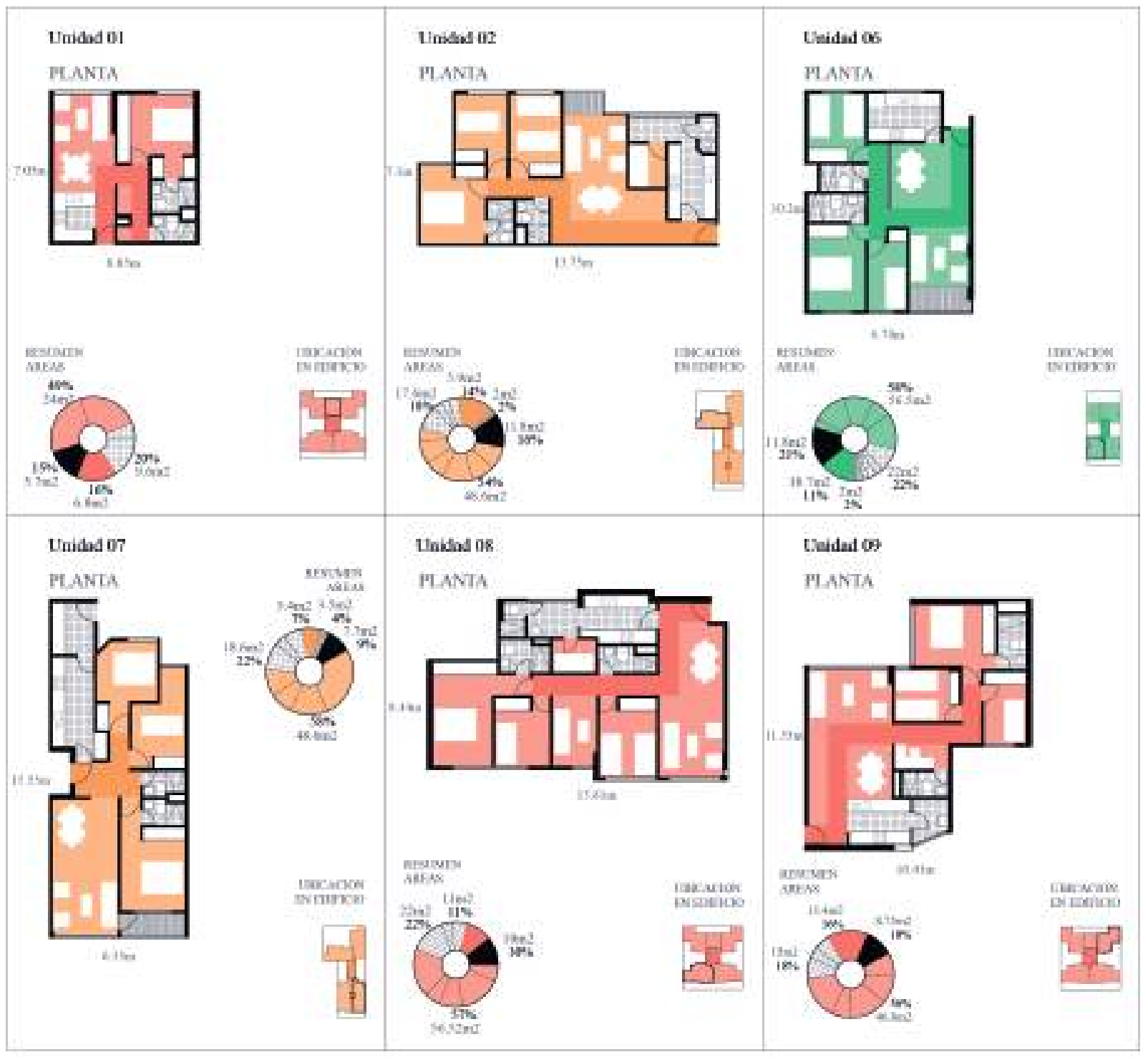

Figura 4

Dibujos en planta a escala de unidades de los casos comentados con sus respectivos gráficos resumen Elaboración propia 
Resumen de áreas. En un gráfico tipo torta se distribuyen los porcentajes relativos destinados a cada tipo de área según las texturas utilizadas en el dibujo (habitaciones, muros, circulaciones, servicios y exteriores). Se complementa cada una indicando los valores absolutos en metros cuadrados. Permite visualizar el grado de optimización o economía de la planta al contraponer las superficies de estancia y distribución.

Semejanza entre ambientes. Pretende visualizar los ambientes en cuanto a su forma y extensión para comparar su jerarquía. "La similitud y regularidad, sumados a un esquema espacial no jerarquizado se entienden como positivos, pues permiten la posible flexibilidad de amueblamiento y uso al interior de la vivienda" (Martín Blas, 2010, p. 5).

Densidad. La intención es aproximarse al espacio útil que tiene que ser repartido y compartido entre los ocupantes de la unidad. $\mathrm{El}$ área habitable expresada en metros cuadrados corresponde a la suma de todas las estancias de la unidad (sala, comedor y habitaciones), excepto el área de muebles fijos. La densidad útil es la relación entre el área habitable y la cantidad de miembros de la unidad de convivencia (metros cuadrados útiles/persona) según el número de camas. La ocupación se refiere al ratio entre la cantidad de personas y el número de habitaciones (personas/habitación). Permite comprobar si cada persona puede disponer de un espacio propio o, en caso de que el valor supere a uno, si es necesario compartir alguna de las habitaciones.

\section{RESULTADOS}

\section{El edificio}

Subdivisión de área libre. Los lotes angostos y profundos obligan, en el caso de edificios multifamiliares, a fragmentar el porcentaje de área libre —entre 30\% y 35\% del área del terreno- en pozos interiores con formas cada vez más irregulares, a fin de cumplir con las distancias mínimas normativas. Sin embargo, por estas mismas razones (pequeñas áreas entre muros cuatro veces su altura), se hace imposible utilizarlas colectivamente a nivel de suelo.

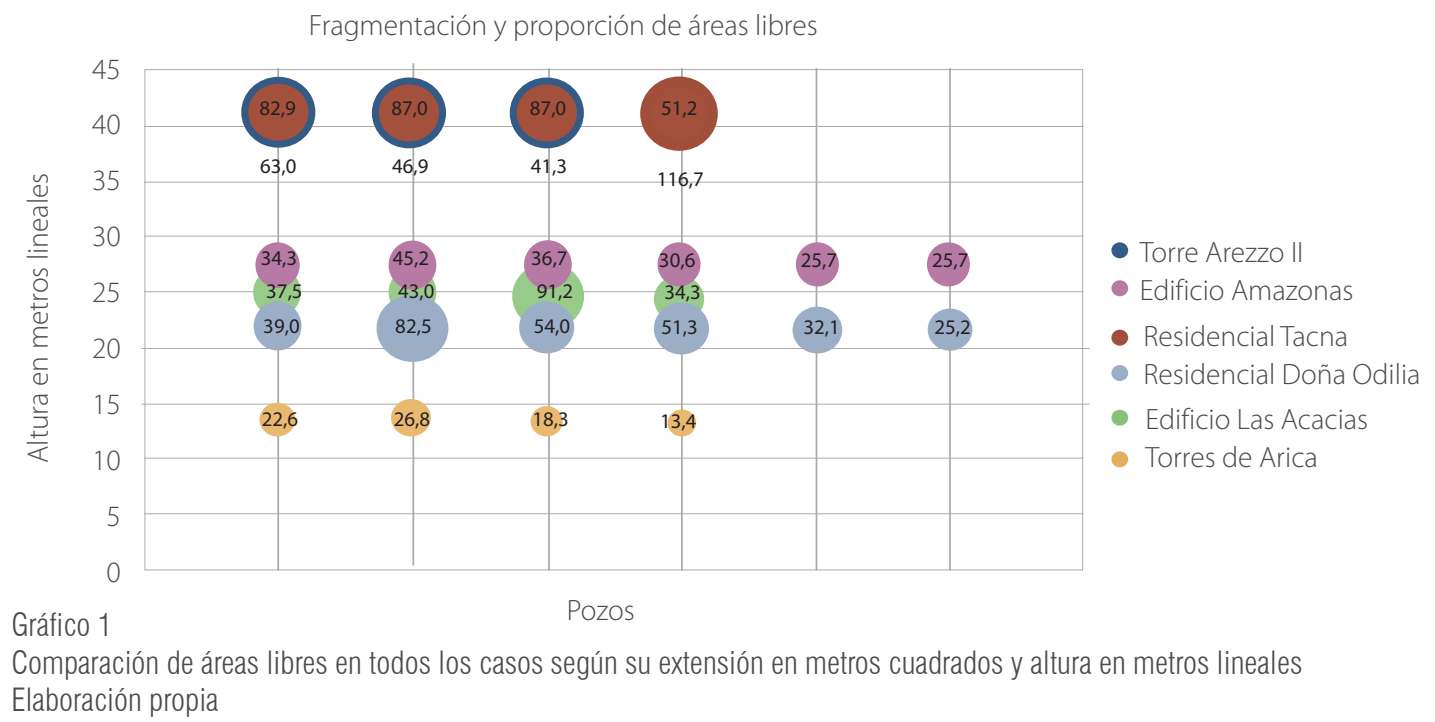

El multifamiliar contemporáneo en la manzana limeña: el caso de Magdalena del Mar, 93-111 
En los casos del edificio Amazonas y el edificio Doña Odilia los pozos son compartidos por varias unidades, comprometiendo incluso la privacidad entre viviendas. Pareciera ser que en los multifamiliares se prioriza el contacto con la calle por sobre la calidad de la relación entre las unidades interiores.

Tipos de unidades. Predomina la orientación doble pasante con los servicios de forma longitudinal o transversal.

\begin{tabular}{|l|cccc|}
\multicolumn{1}{|c|}{ Edificio } & Simple & Pasante & Dúplex & Esquina \\
\hline Torre Arezzo II & 42 & 28 & 0 & 0 \\
Residencial Tacna & 26 & 28 & 2 & 0 \\
Edificio Las Acacias & 0 & 32 & 4 & 0 \\
Edificio Amazonas & 0 & 50 & 0 & 0 \\
Residencial Doña Odilia & 7 & 28 & 5 & 0 \\
Torres de Arica & 0 & 12 & 3 & 0 \\
\hline TOTAL & 75 & 178 & 14 & 0
\end{tabular}

Tabla 1

Conteo de los tipos de unidades en todos los casos

Elaboración propia

Lo más llamativo son las subvariantes que, de distintas maneras (escalonamientos y recesos), buscan minimizar el frente a expensas de una gran profundidad siguiendo las proporciones del lote, como sucede con el edificio Amazonas y la residencial Tacna. La consecuencia más legible es la presencia de la simetría bilateral en la fachada por la presencia de un par de unidades pasantes. Su repetición no es en paralelo como sucede en los edificios tipo bloque o hilera, sino hacia el interior del lote, enfrentando sus caras libres. En consecuencia, este ensamble va en contra de las lógicas del tipo mismo.
Agrupación y acceso. Los ascensores directos funcionan para dos unidades contiguas como máximo. Sin embargo, por su elevado costo, a mayor número de núcleos se reduce la economía del edificio (residencial Tacna). El ratio entre unidades/núcleos es una de las principales dificultades por resolver en los edificios en lotes típicos, ya que la eficiencia de la circulación común es usualmente correlativa a los edificios compactos como Torre Arezzo II. La solución que observamos en la mayoría de los edificios es mantener un solo núcleo para acceder a múltiples unidades a través de un 
corredor común extenso, a costa de la dispersión de su huella. En el caso de Torres de Arica, la estrechez del lote obliga a colocar tres unidades una detrás de otra. El ratio de accesos es el más bajo, ya que un núcleo sirve a dos unidades y el otro solo a una unidad, como consecuencia de las condicionantes propias del lote y la edificabilidad señalada en los parámetros.

Cerramiento perimétrico. Se trata de edificios en los que siempre la extensión de las fachadas exteriores es menor que las fachadas interiores y los frentes ciegos.

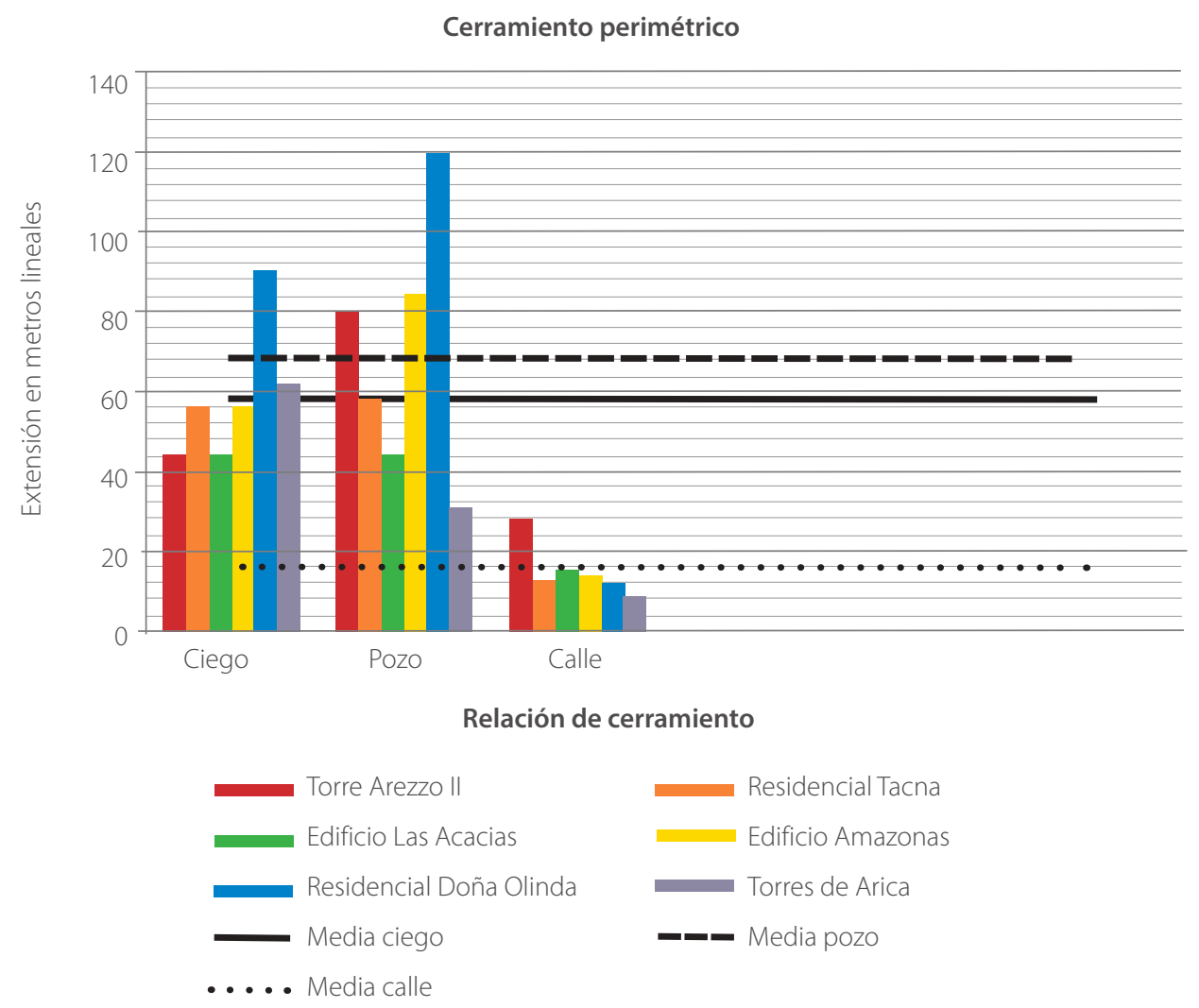

Gráfico 2

Extensión en metros lineales de los casos de estudio según tipos de envolventes Elaboración propia 
Incluso en los edificios más compactos como Las Acacias y Torre Arezzo II, los frentes ciegos son inevitables por tratarse de una arquitectura de lote, es decir, edificios confinados entre muros medianeros sin normas que eviten el contacto lateral (pero sí obligan a retiros frontales). Los inconvenientes de esta condición son mayores en los edificios de menor frente y mayor profundidad, ya que la complicidad entre la fragmentación de vacíos y la cantidad de circulaciones comunes trae consigo edificios dispersos donde los frentes ciegos constituyen más del 50\% de su envolvente.

Relación con la calle. En cuatro de los seis edificios dentro del área de estudio, se prioriza al auto sobre el peatón, es decir, se destina la mayor cantidad de frente a rampas de ingreso vehicular o estacionamientos.

Relación con la calle

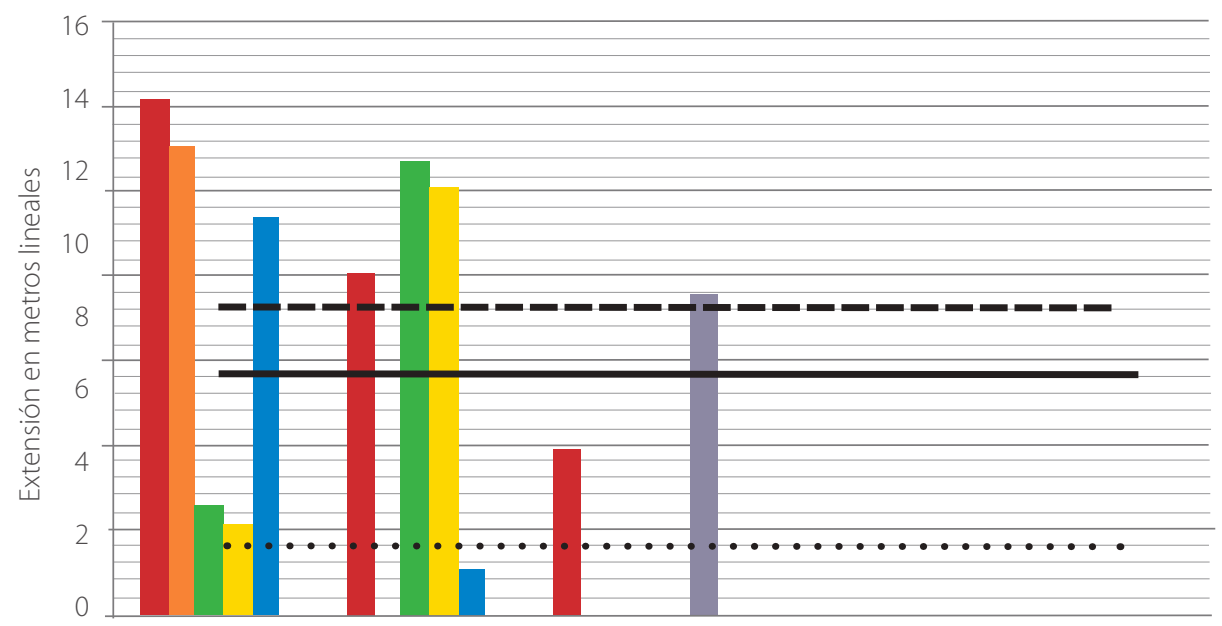

Ciego

Permeable accesible

Tipo de cerramiento
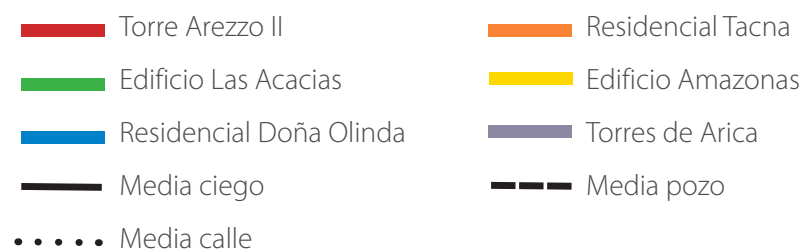

Gráfico 3

Extensión en metros lineales de los casos de estudio según tipos de cerramiento hacia la calle Elaboración propia 
La residencial Doña Odilia es una excepción, pero evita abrirse a la calle mediante un muro opaco. Solo Torres de Arica prescinde de un cerramiento a plomo de vereda. La exigencia de estacionamientos por vivienda hace que, para un edificio de ocho pisos en un lote típico, al menos $6 \mathrm{~m}$ de los $15 \mathrm{~m}$ de fachada se destinen a rampas vehiculares, es decir, el $40 \%$ del frente a la calle. El retiro visto desde esta problemática solo fomenta el divorcio entre el edificio y la calle, ya que los estacionamientos ahí ubicados maximizan su eficiencia en vez de construir una relación visual y de animación con la calle, lo que probablemente resultaría más seguro que negar este contacto.

\section{LA UNIDAD}

\section{Resumen de áreas}

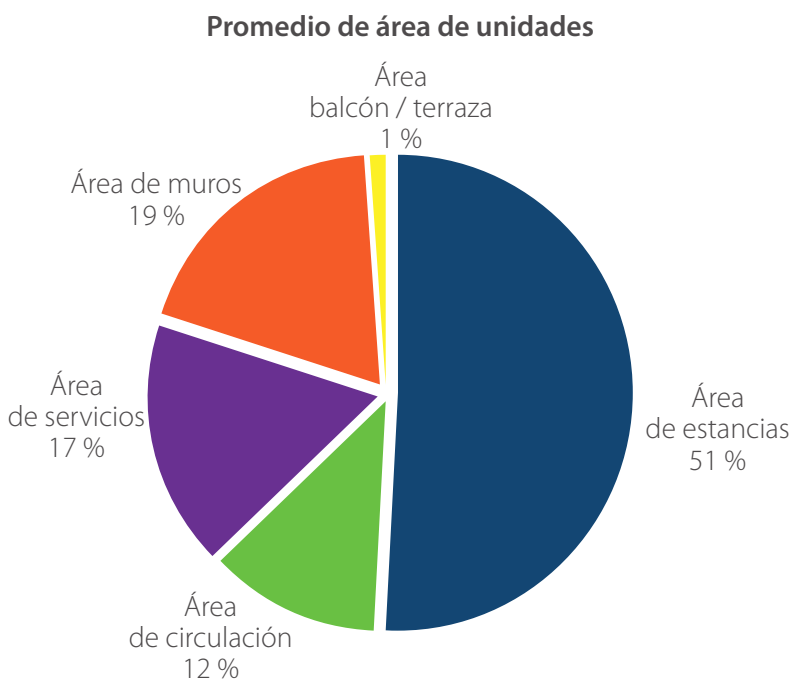

Gráfico 4.

Esquema tipo torta con el promedio de tipos de áreas Elaboración propia
La medición de áreas muestra en las unidades analizadas un porcentaje de circulación por encima del $10 \%$. Según su distribución interior las unidades se pueden clasificar en distintos tipos: corredor, hall, núcleo, patio, escalera, racimo (Martín Blas, 2011, p. 11). En los ejemplos predomina el tipo corredor, ingresa por la sala comedor (incluso la atraviesa), luego pasa por la cocina y cada una de las habitaciones. Las unidades de $90 \mathrm{~m}^{2}$ con recorridos tortuosos son las de mayor porcentaje de circulación (unidad 9). En unidades con acceso por un vestíbulo o punto intermedio (unidad 7) se reduce la distancia por recorrer, con la posibilidad de elegir ir a la sala comedor o a las habitaciones sin la obligación de atravesar ambientes sociales. Los porcentajes de área de servicio apenas llegan al $20 \%$, pues no hay habitación de servicio (a excepción de las unidades 2 y 8 , aunque se cuentan como estancias), tampoco grandes lavanderías, ni baño de visita o servicio, y el segundo baño usualmente se comparte entre las habitaciones secundarias. Sin embargo, cuando hay que resolver formas peculiares o muy extensas, los ambientes de servicio aparecen dispersos (unidad 11). Esto conlleva a un mayor costo de instalaciones y una menor posibilidad de modificaciones dentro de la unidad.

\section{Semejanza de ambientes}

Tanto las zonas sociales como una de las habitaciones tienen una jerarquía claramente superior. Estos ambientes son también en muchos casos aquellos en donde empieza y termina el recorrido interno (unidades 2, 8 y 15). A su vez, el que usualmente sean ubicados hacia el frente de calle en las unidades pasantes subraya esta valoración. Por otro lado, ya que casi todas las unidades dentro del área de estudio carecen de zonas de estar privadas, las 
habitaciones suelen compartirse y solo albergan camas, y la sala comedor se vuelve un ambiente de uso permanente por necesidad más que por elección. Si tomáramos como referencia que cualquier estancia puede ser considerada como tal si cuenta con al menos $6 \mathrm{~m}^{2}$ libres de elementos fijos (Martín Blas, p. 5), varios de estos ambientes no podrían contar para el cálculo de densidad (unidad 6,11 y 18) cuando se excluye la profundidad del clóset $(60 \mathrm{~cm})$.

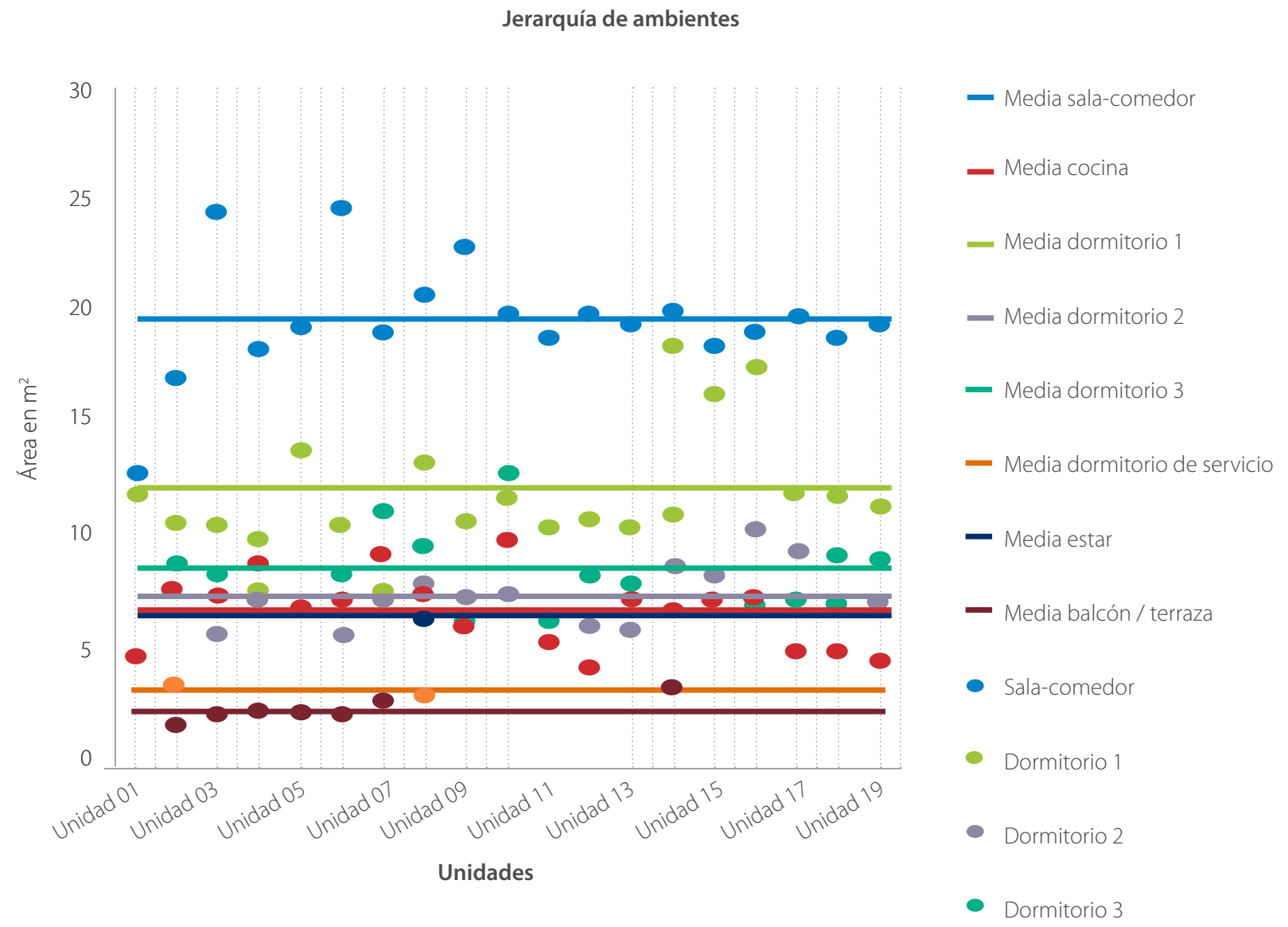

Tabla 2

Matriz con los tamaños y tipos de habitaciones de todos los casos y la media

Elaboración propia 


\section{Densidad}

El área habitable reduce prácticamente a la mitad el área nominal de todas las unidades. Como el área y la cantidad de los servicios se mantienen casi igual entre unidades de dos o tres dormitorios, la densidad útil aumenta, es decir, a cada persona le corresponden menos metros cuadrados. Tal es el caso de la unidad 1, de un dormitorio y $14,44 \mathrm{~m}^{2} /$ persona, a diferencia de la unidad 15 , de dos dormitorios y de $11,33 \mathrm{~m}^{2} /$ persona, y finalmente la unidad 11 con una densidad útil de $7,39 \mathrm{~m}^{2} /$ persona. Esta observación se hace menos deductiva al constatar que los parámetros suelen normar los tipos de unidades en relación a su área y cantidad de dormitorios, pero la diferencia entre un departamento de un dormitorio y otro de tres en metros cuadrados pude ser de $20 \mathrm{~m}^{2}$, es decir, el área de dos habitaciones. Otro factor más objetivo para cuantificar la calidad o la exclusividad de las viviendas es la cantidad de ambientes y servicios que hay que compartir. ¿Qué ocurre cuando no hay baño de visita, por ejemplo? Para utilizar el baño secundario es necesario ingresar a la zona privada, de forma que se trastoca la jerarquía rígida de la vivienda (unidad 18), e incluso atenta contra la propia organización tipológica. La sala de estar es una estancia comodín que puede aumentar o disminuir la densidad, dependiendo de la manera en que el diseño resuelve sus relaciones al interior de la unidad y con el exterior. En la unidad 14, el estar es una estancia que aumenta los metros cuadrados/persona a $11,86 \mathrm{~m}^{2}$, aunque la ocupación se mantiene en dos personas/habitación. Pero por su mala ubicación estratégica - hacia el pozo interior y por el lado más corto enfrentando al vecino-, dificilmente puede ensamblarse para darle un uso distinto a esa estancia. Caso contrario sucede con el estar de la unidad 8, que podría transformarse en un dormitorio dentro de sus propias lógicas. Esta unidad se apega al tipo de un solo frente y corredor de manera más neutral y directa.

\section{DISCUSIÓN Y CONCLUSIONES}

El diseño de edificios multifamiliares bajo la premisa del trazado urbano limeño, dirigido por los parámetros normativos distritales y regulado por el Reglamento Nacional de Edificación (RNE), refuta los criterios con que se estudia y se proyecta la vivienda colectiva. Como hemos visto, hoy en día las manzanas en el distrito de Magdalena se (des)componen mediante un tipo de multifamiliar cuya repetición no contempla la voluntad de generar un tejido urbano coherente.

En cuanto a las unidades de vivienda, el análisis nos permite concluir que los futuros cambios demográficos de la sociedad dejarán obsoleta una estructura tipológica heredada de la clásica familia nuclear y jerárquica del siglo $\mathrm{xx}$, incapaz de adaptar un concepto de familia que se encuentra invariablemente a merced de los nuevos contextos sociales. En su lugar, habría que dar respuesta a diversos subgrupos de convivencia: familias monoparentales, parejas sin hijos, parejas homosexuales, independientes que trabajan en casa, entre tantos otros.

Por otro lado, la mayor movilidad social de la nueva clase media pondrá en jaque la rigidez de las viviendas que parecen estar dirigidas a un momento específico de la misma familia. Con densidades útiles por debajo de los $10 \mathrm{~m}^{2}$ por persona, habitaciones que solo sirven para dormir, zonas sociales que deben compartirse por obligación y contradicciones en usos y recorridos, es probable que estas unidades sean disparadoras de conflictos internos cuando 
los miembros de estas familias exijan un nivel distinto de convivencia en el futuro. Sostenemos que las unidades que respetan los esquemas tipológicos más esenciales, paradójicamente, consiguen la flexibilidad que las condiciones cambiantes de toda convivencia demanda.

A escala del edificio comprobamos que la agrupación de estas unidades tampoco es capaz de proponer modelos de vida colectiva coherentes. Sometidos a un mercado, normativas y lotización asfixiantes, el gran porcentaje de área libre se fragmenta en pozos inutilizables, los tipos se desfiguran y dejan de trabajar en conjunto, a la vez que las agrupaciones se contradicen con las unidades, cuyo resultado son las volumetrías dispersas y de muros ciegos. Los tipos de edificios suelen definirse por la forma en que se agrupan sus unidades y se accede a ellas: bloque, manzana, torre, hilera. La peculiaridad de este aspecto de la mayoría de edificios en el área de estudio hace que escapen a estas categorías comprobadas.

Por sus características y atributos tipomorfológicos, definimos los casos estudiados bajo un prototipo de edificio recurrente que resulta de estas condicionantes particulares: el tren. El tipo tren hace alusión a los edificios cuya planta se resuelve con un corredor central extenso a lo largo del cual se suceden unidades de vivienda pasantes en pares, uno tras otro separados por pozos internos para iluminar la parte media y más profunda de su huella hasta llegar al fondo del lote. A pesar de sus deficiencias, podemos afirmar que por su persistencia —en términos de Lee- es ya un tipo dominante limeño, es decir, encarna los principios estructurales, organizacionales, sociales y económicos de nuestra ciudad. Podemos reconocer, por la simetría bilateral en fachada y la sucesión de enormes muros medianeros ciegos, que esta resolución particular del tipo tren no está limitada a los edificios dentro de la zona de estudio y que persiste en muchos de los entornos urbanos de Lima. Por lo tanto, preocupa que los resultados y observaciones planteados puedan ser directamente extrapolables a tantas otras zonas de la ciudad.

\section{UNA VISIÓN DE CIUDAD}

Partimos de la premisa que la manzana debe proyectarse sobre la base de una idea colectiva y, luego, teniendo en cuenta el papel asignado a cada lote. De esta forma, cada edificio podrá construirse en diferentes momentos, gestionado por distintos inversionistas y diseñado por varios arquitectos, pero siempre será parte de un conjunto mayor premeditado. Es necesario reconocer que los lotes son enormemente variados y no están aislados.

Actualmente, hay lotes de determinados tamaños condenados a albergar un tipo de edificio multifamiliar deficiente que será explotado al máximo. La especulación sumada a una zonificación ajena a esta realidad limita la posibilidad de integrarse o relacionarse de forma positiva y generosa con el contexto inmediato. Como hemos visto, las consecuencias morfológicas de este fenómeno son los perfiles urbanos incompletos, discontinuos y de muros ciegos que tipifican estos entornos. Existe una contradicción inherente entre el tipo de edificio que promueven los parámetros y su situación urbana, pues la única alternativa compacta para resolver tan altas densidades en altura es la torre de núcleo central. Y es justamente en este tipo - en el que los ambientes utilizan todo el perímetro para ventilar e iluminar- que se puede llegar incluso a prescindir de frentes ciegos. 
Habría que rescatar el concepto de edificabilidad (metros cuadrados edificables/metros cuadrados de terreno) como un valor para toda la manzana. De esta manera, un coeficiente determina la capacidad de edificar sobre una superficie general, que se pueda modelar en conjunto de acuerdo a las posibilidades tipológicas de cada lote. Los lotes angostos y profundos son capaces de soportar un número finito de tipos hasta cierta altura y densidad, como el tipo quinta. Colmatar la edificabilidad a esta escala daría lugar a la heterogeneidad en las propias manzanas, principio que creemos necesario dentro de las condicionantes que presenta la diversidad de lotes limeños.

Del mismo modo podemos pensar en la posibilidad de consolidar el porcentaje de área libre de manera conjunta para toda la manzana. ¿Por qué limitar la obligación de llegar al tercio de la altura dentro del lote propio, y no de forma compartida entre lotes de modo que cada edificio se retira de la medianera un sexto de su altura y a la vez gana un frente libre? Además del aprovechamiento compartido de las dimensiones con unidades interiores que miren a espacios amplios en vez de pozos, también este porcentaje se puede proyectar como superficies de uso colectivo al interior de la manzana.

Toda visión alternativa de ciudad conlleva, indefectiblemente, tipos de unidades y por tanto de edificios específicos para su correcto funcionamiento en el sentido más amplio del término. En el ámbito de la vivienda, los tipos comprobados son parte fundamental del proceso de diseño. Esto no impide planteamientos novedosos, como sostiene Moneo, la reflexión tipológica supone todo lo contrario.
Así como hace doscientos años las manzanas de Idelfons Cerdá en Barcelona fueron (r)evolucionarias, hoy en día son el fondo que hace de sus entornos urbanos uno de los más reconocibles en Europa. Experiencias recientes como el open block en el Rive Gauche de París (Accorsi, 2010) son ensayos propositivos con un enfoque contemporáneo de manzanas permeables y heterogéneas. Creemos que Lima tiene pendiente encontrar esa huella genética propia. Las ideas y criterios tipo-morfológicos aquí esbozados pretenden pensar los edificios multifamiliares no de manera fragmentada y hermética, sino dentro de un tejido enhebrado por piezas capaces de dialogar entre sí para proponer múltiples configuraciones.

\section{REFERENCIAS}

Accorsi, F. (2010). The Open Block: Christian de Portzamparc. Bruselas: Archives d'Architecture Moderne.

Gonzales de Olarte, E., Del Solar,V., y Del Pozo, J. M. (2011). "Lima metropolitana después de las reformas neoliberales: transformaciones económicas y urbanas". En C. De Mattos, W. Ludeña, y L. Fuentes. (Eds.) (2011). Lima_Santiago: reestructuración y cambio metropolitano. Lima: Pontificia Universidad Católica del Perú, Centro de Investigación de la Arquitectura y la Ciudad;Santiago de Chile: Pontificia Universidad de Chile, Instituto de Estudios Urbanos y Territoriales.

Heckmann, O., y Schneider, F. (Eds.) (2011) Floor plan housing manual (3. ${ }^{a}$ ed.). Basilea: Birkhauser. 
Jacoby, S., y Lee, C. (Eds.) (2007) Typological Formations: Renewable Building Types and the City. Londres: AA Publications.

Kahatt, S. (2015). Utopías construidas. Las unidades vecinales de Lima. Lima: Pontificia Universidad Católica del Perú.

Kaijima, M., Kuroda, J., y Tsukamoto, Y. (2008) Made in Tokyo. Tokio: Kajima Institute Publishers.

Kempe, A., y Thill, O. (2004). "Neutralidad específica. Un manifiesto sobre la nueva vivienda colectiva". En J. Mozas, A. Fernández-Per (2004) Densidad: nueva vivienda colectiva. Vitoria-Gasteiz: $\mathrm{a}+\mathrm{t}$ ediciones.

Koolhaas, R. (2004 [1978]) Delirio de NuevaYork. Un manifiesto retroactivo para Manhattan. Barcelona: Gustavo Gili.

Lassance, G., Varella, P., y Costa-Capille, C. (2013) Rio Metropolitano. Río de Janeiro: Rio Books.

Ledgard, R. (1987). La Ciudad Moderna. En: Ledgard, R. (2015). La ciudad moderna. Textos sobre arquitectura peruana. Lima: Pontificia Universidad Católica del Perú, Fondo Editorial, pp. 137-155.

Ledgard, R., y Solano, A. (2011). "Lima: transformaciones en la estructura e imagen de la metrópoli: situación actual y perspectivas" En: C. De Mattos, W. Ludeña, y L. Fuentes. (Eds.) (2011).Lima_Santiago:reestructuración y cambio metropolitano. Lima: Pontificia Universidad Católica del Perú, Centro de Investigación de la Arquitectura y la Ciudad;
Santiago de Chile: Pontificia Universidad de Chile, Instituto de Estudios Urbanos y Territoriales.

Leupen, B., y Mooij, H. (2011). Housing Design: A Manual. Róterdam: nai010 publishers.

Ludeña, W. (mayo del 2002). Lima: poder, centro y centralidad: Del centro nativo al centro neoliberal. EURE (Santiago)-Revista Latinoamericana de Estudios Urbanos Regionales, (83), 45-65.

Ludeña, W. (mayo del 2006). Ciudad y patrones de asentamiento. Estructura urbana y tipologización para el caso de Lima. EURE (Santiago)-Revista Latinoamericana de Estudios Urbanos Regionales, (95), 37-59.

Ludeña,W. (diciembre del 2006). Barrio y ciudad Historiografia urbanística y la cuestión del dominio de referencia. El caso de Lima. Bitácora Urbano-Territorial (Bogotá), (10), 82-105.

Ludeña,W. (2011) Lima: transformaciones urbanas y reestructuración morfológica. Urbanismo, vivienda y centro histórico. Periodo 19902007. En C. De Mattos, W. Ludeña, y L. Fuentes. (Eds.) (2011). Lima_Santiago: reestructuración y cambio metropolitano. Lima: Pontificia Universidad Católica del Perú, Centro de Investigación de la Arquitectura y la Ciudad; Santiago de Chile: Pontificia Universidad de Chile, Instituto de Estudios Urbanos y Territoriales.

Martín Blas, S. (2011) I+D+VS Futuros de la vivienda social en 7 ciudades. Madrid: Fundación Arquitectura COAM. 
Moneo, R. (1978). On Typology. En Oppositions 13. Massachussetts:The MIT Press, 23-45.

Mozas, J., y Fernández-Per, A. (2004) Densidad, Nueva vivienda colectiva.Vitoria-Gasteiz: $\mathrm{a}+\mathrm{t}$ ediciones.

Pérez de Arce, R. 2012. Domicilio urbano. Santiago: Ediciones ARQ.

Rossi, A. (1999 [1971]). La Arquitectura de la ciudad (10. ${ }^{a}$ ed.). Barcelona: Gustavo Gili.
Sherwood, R. (1994). Modern housing prototypes (6. ${ }^{\mathrm{a}}$ ed.). Cambridge, London: Harvard University Press.

Venturi, R., Izenour, S. y Scott-Brown, D. (2004 [1972]) Aprendiendo de Las Vegas, el simbolismo olvidado de la forma arquitectónica (5. ${ }^{a}$ ed.) Barcelona: Gustavo Gili. 
